\title{
LA-UR-14-27033
}

Approved for public release; distribution is unlimited.

Title: Stand-Alone Dynamic System Simulation of Fissile Solution Systems

Author(s): $\quad$ Klein, Steven Karl

Determan, John C.

Dowell, Larry Jonathan

Roybal, Marsha Marilyn

Intended for: Report

Issued: $\quad$ 2014-09-10 (rev.1) 
Disclaimer:

Los Alamos National Laboratory, an affirmative action/equal opportunity employer,is operated by the Los Alamos National Security, LLC for the National NuclearSecurity Administration of the U.S. Department of Energy under contract DE-AC52-06NA25396. By approving this article, the publisher recognizes that the U.S. Government retains nonexclusive, royalty-free license to publish or reproduce the published form of this contribution, or to allow others to do so, for U.S. Government purposes. Los Alamos National Laboratory requests that the publisher identify this article as work performed under the auspices of the U.S. Departmentof Energy. Los Alamos National Laboratory strongly supports academic freedom and a researcher's right to publish; as an institution, however, the Laboratory does not endorse the viewpoint of a publication or guarantee its technical correctness. 


\section{Stand-Alone Dynamic System Simulation of Fissile Solution Systems}

Determan, John C., Dowell, Jonathan, Klein, Steven K., \& Roybal, Marsha M.

September, 2014

References 1 through 6 document a family of dynamic system simulation models of fissile solution systems. Historical aqueous homogeneous reactors (AHR) modeled include: SUPO (Super Power), KEWB (Kinetics Experiment Water Boiler) " $A$ " and "B" cores, Silene, and HRE (Homogeneous Reactor Experiment) that are considered benchmarks for operational characteristics of fissile solution systems. All operating modes of these systems are examined in the models including steady-state, delayed critical, prompt critical, and boiling. Model results are compared with experimental data for each AHR to demonstrate the validity of the modeling technique employed.

\section{Modeling Environment}

Dynamic system simulations to date have been constructed utilizing Direct-Executing Simulation in Real Time (DESIRE) ${ }^{1}$ on IBM-compatible personal computers. The DESIRE modeling technique is a powerful tool for scientific computation in a direct runtime environment allowing the investigator to avoid costly and time consuming use of mainframe or distributed cluster computing systems. However, DESIRE modeling does require facility with Korn DESIRE scripting and availability of the corresponding software package.

This Report describes the conversion of two specific models of fissile solution systems, SUPO and a generic accelerator-driven system, to a stand-alone executable program. This conversion utilized Microsoft Visual Studio as the software development tool and thus is compatible with desktop or server hosted platforms running Microsoft Windows.

\section{Functionality}

The DESIRE modeling technique allows complete user access to all model structures. This includes input constants, variables, execution and display features. Mathematical expressions similarly can be directly modified by the user thereby maximizing simulation flexibility. However, this universal access to model coding opens the prospect of departing from validated system models of historical AHR.

It is recognized that potential users of these models exist whose interest lies with system operations rather than underlying numerical analysis and modeling techniques. It is for this group of the nuclear engineering community that the stand-alone versions have been developed.

\footnotetext{
${ }^{1}$ Interactive Dynamic-System Simulation, Granino Korn, October 21, 2010 
User access to the stand-alone simulation models is via a graphical user interface (GUI). The GUI provides the ability to modify certain physical, configuration, and operational parameters of the system. Underlying equations-of-state for neutron transport, thermal hydraulics, radiolytic gas generation and transport, plenum dynamics and other parameters effecting core performance are not accessible. What has been retained is essentially the full suite of parameters that could be modified by a system operator. In essence the user may examine system performance of a variety of real-world parameters. Inserted reactivity and rate of insertion, coolant flow rate and initial temperature, and fuel initial temperature are examples.

Control over simulation duration as well as display scaling is also available. This allows the user to study such phenomena as initial system power pulse, and transition to steady-state, including initial fuel temperature rise and onset of radiolytic gas saturation in the core.

\section{User Interface}

Figure 1 is the user interface for the SUPO model.

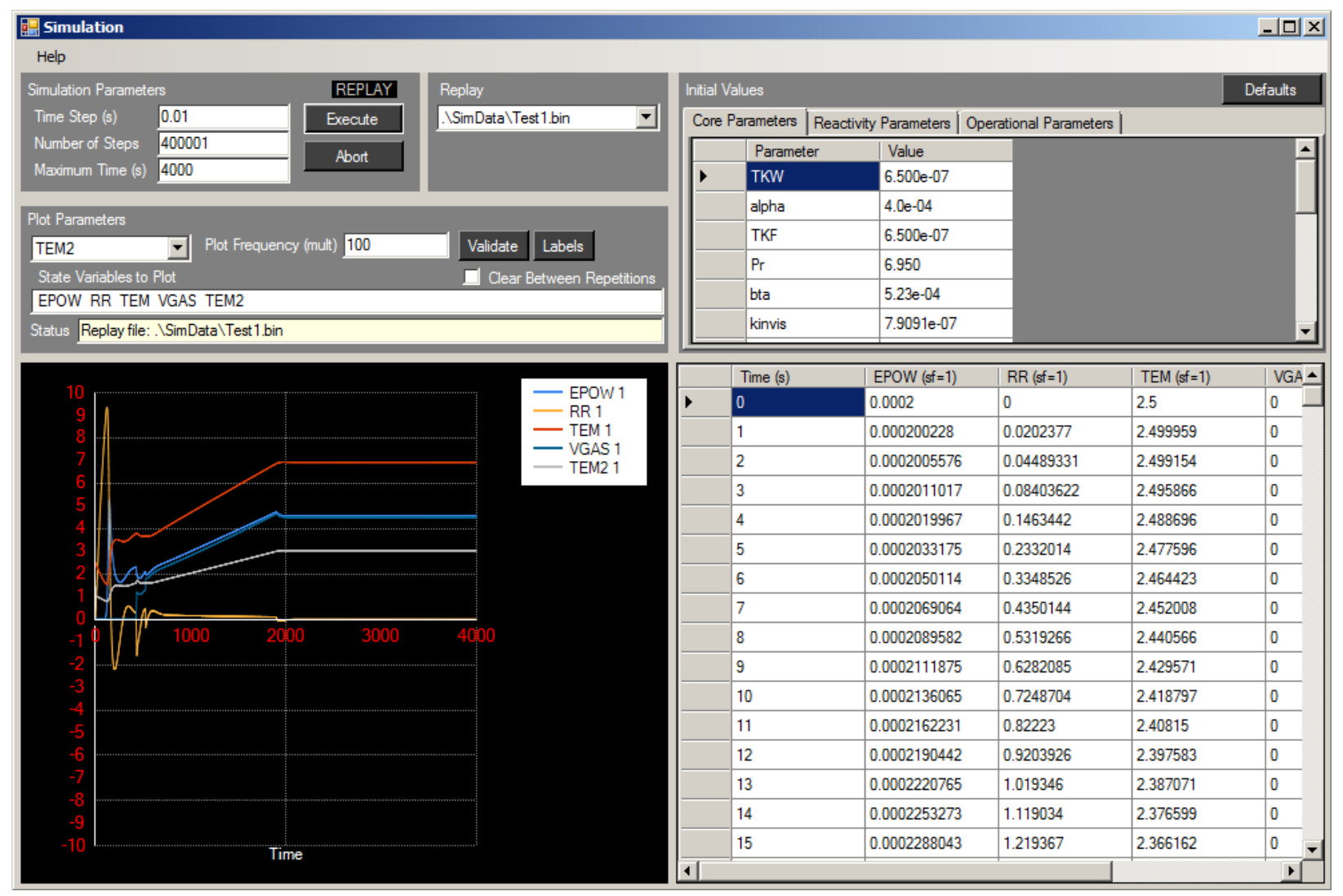

Figure 1: Graphical Users Interface for SUPO Dynamic System Simulation

Four regions are visible in the display. The upper panels are devoted to setting simulation parameters and execution while the lower panels display the output of the simulation. The physical parameters of 
the simulation controllable by the user are located in the upper right corner of the interface and are accessible by three tabs: Core, Reactivity, and Operational Parameters.

Core parameters generally refer to physical constants of the fuel itself, core height and volume, and those having to do with the thermal hydraulic characteristics of an in-core forced cooling system composed of cooling coils as was the case with SUPO. Reactivity parameters include flux profile and importance factors by core region, reactivity feedback due to radiolytic gas void and fuel temperature, delayed neutron fraction and average transit time for a radiolytic gas bubble to escape the core. Operational parameters include initial fuel and coolant temperatures, coolant flow rate, plenum pressure, reactivity to be inserted, rate of reactivity insertion and point-in-time of the insertion, and initial core reactivity.

The simulation control panel provides user choice over maximum time of the simulation and associated numerical analysis attributes and parameters for graphical plotting. Certain verification and validation tools are also provided to improve success of execution. Control to rerun a previously saved model is also provided. The lower panels of the interface provide graphical output of the parameters chosen for display and an adjacent database of values stored in memory for those parameters.

\section{DESIRE versus Visual Studio}

A detailed comparison of output parameters between the two implementations confirms the precision of the conversion process, which was expected in that the process does not include specific recoding of any mathematical expressions. Essentially a converter has been developed, which translates DESIRE script into corresponding $\mathrm{C}$ code. This approach allows retention of the underlying execution of the mathematical expressions without modification. Certain information such as constant and variable declarations must be created, but syntactically converted code is otherwise placed in appropriate routines depending on whether the code should be executed once (initialization) or in a loop (numerical integration). The separate, but linked, GUI is additional capability and is wholly new code implemented in the C-version. Specifically, the dynamic simulation was written using Visual $\mathrm{C}++$, and the GUI using $\mathrm{C \#}$. The model converter program was written in $\mathrm{C \#}$. The converter allows the rapid translation of any dynamic system simulation developed in DESIRE to a Visual Studio version for interested users.

Figure 2 presents the DESIRE output of the simulation of SUPO with the same input parameters as those presented in Figure 1 using the Visual Studio implementation. 


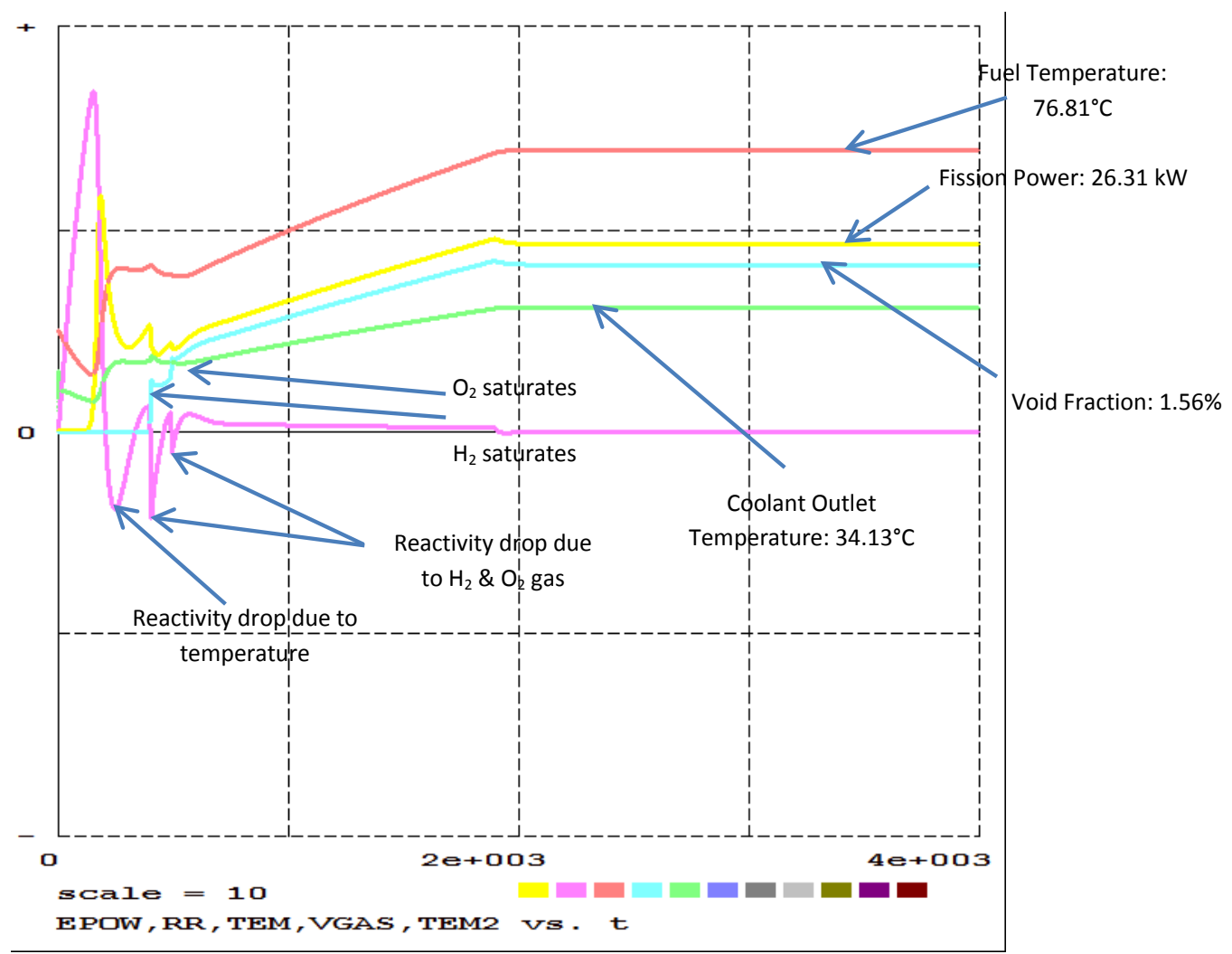

Figure 2: DESIRE Implementation of SUPO Simulation

\section{Accelerator-Driven Systems}

Figures 3 and 4 present the Visual Studio and DESIRE outputs for a generic accelerator-driven system utilizing fissile solution fuel; note the modifications in the Visual Studio users interface. This modification has been performed to give the interface more of a "console feel" in that the various controls and displays are typical of the arrangement of that found on an instrumentation and control system. As in the SUPO implementation described previously, comparison of detailed results illustrates the precision of the conversion. 


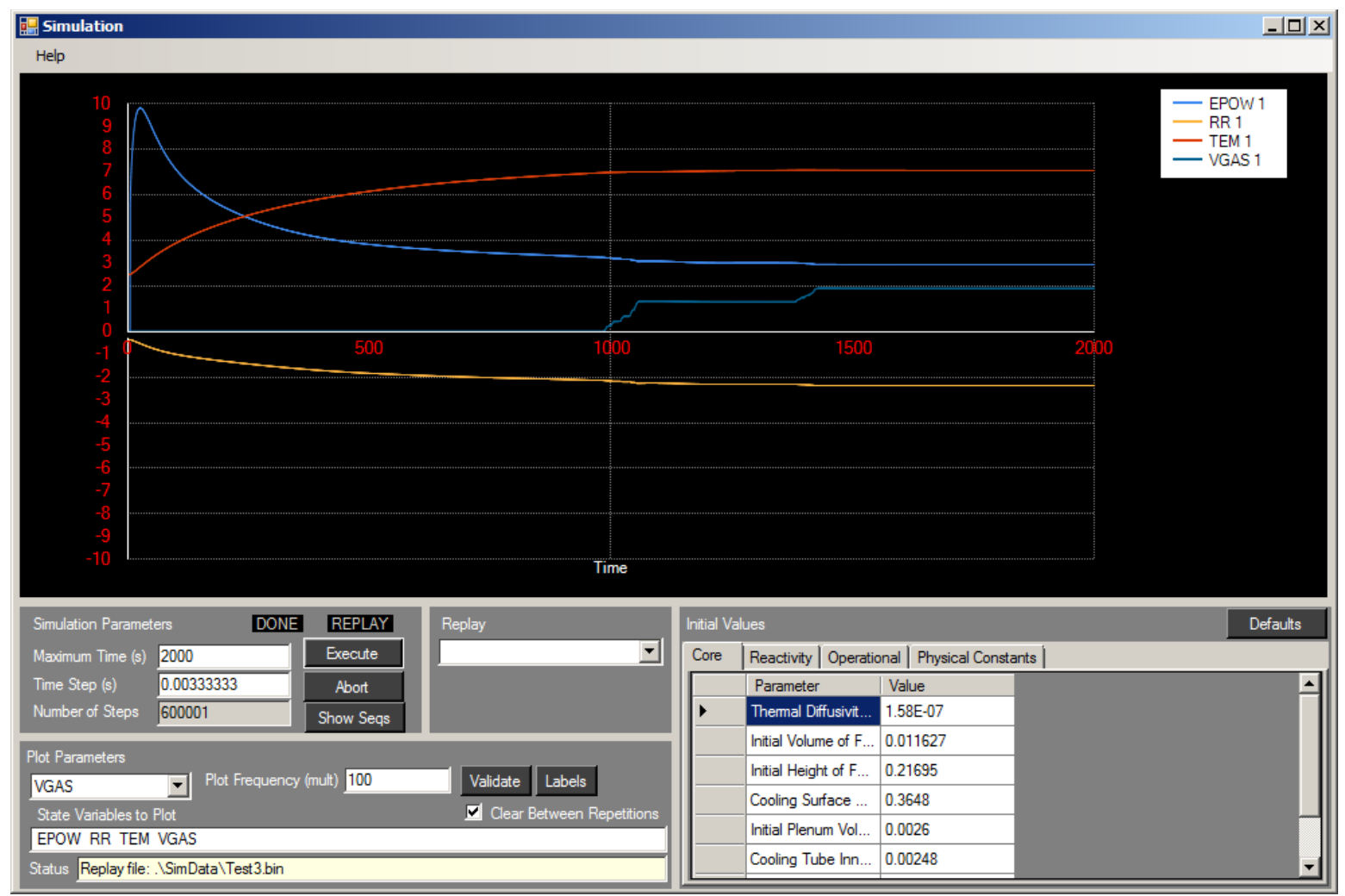

Figure 3: Graphical Users Interface for Accelerator-Driven System Simulation

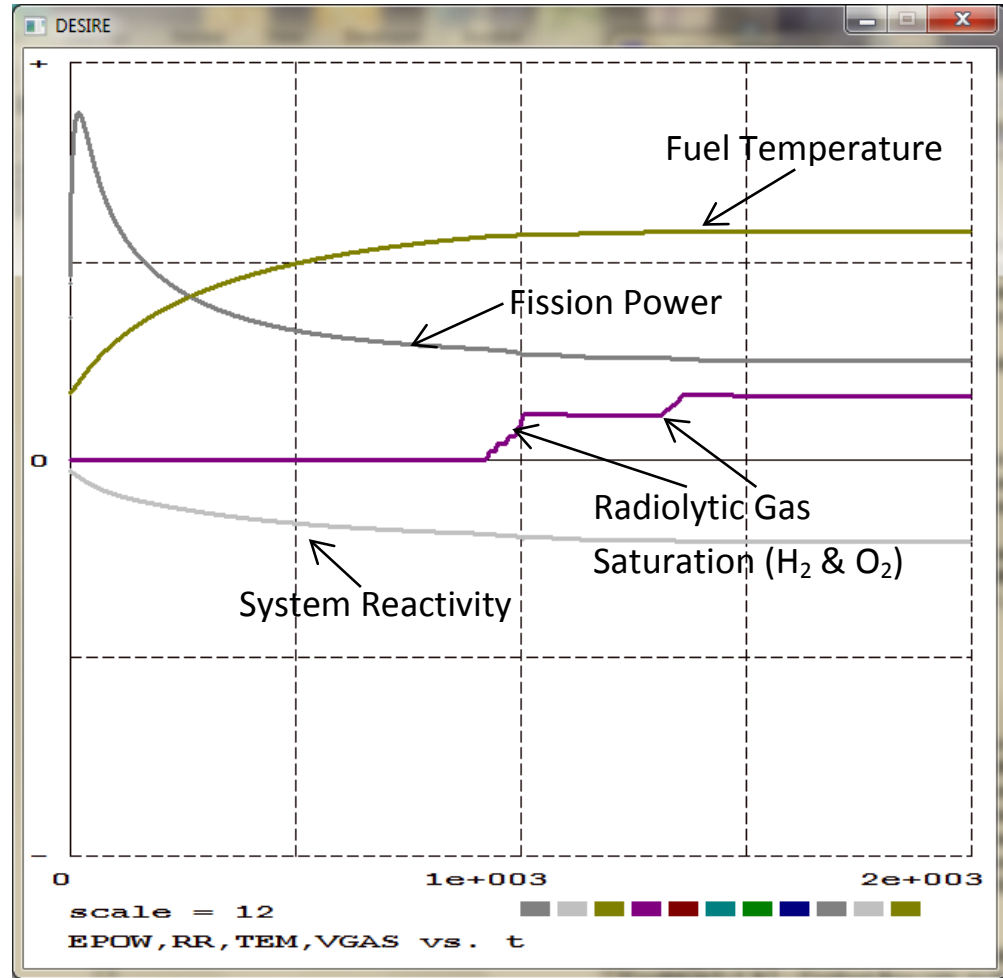

Figure 4: DESIRE Implementation of Simulation of an Accelerator-Driven System 


\section{Summary}

The utility and precision of the conversion of a dynamic system simulation prepared in DESIRE to Visual Studio has been accomplished. The Visual Studio implementation provides a user interface, which may be used for a variety of purposes including training and evaluation of the operational behavior of these systems.

\section{References}

1. A Generic System Model for a Fissile Solution Fueled System, LA-UR-13-22033; Kimpland, Robert H. \& Klein, Steven K., July, 2013

2. Neutron Diffusion Model for Prompt Burst Simulation in Fissile Solutions, LA-UR-13-26779; Kimpland, Robert H. \& Klein, Steven K., August, 2013

3. A Generic System Model for a Fissile Solution Fueled Assembly - Part II, LA-UR-13-28572; Kimpland, Robert H. \& Klein, Steven K., January, 2014

4. Discussions Regarding the Characteristics of Fissile Solution Systems, LA-UR-14-22661; Kimpland, Robert H. \& Klein, Steven K., March, 2014

5. Discussion Regarding Aqueous Homogeneous Reactor (AHR) Benchmarks, LA-UR-14-23768; Kimpland, Robert H. \& Klein, Steven K., May, 2014

6. Dynamic System Simulation of Fissile Solution Systems, LA-UR-14-22490; Kimpland, Robert H., Klein, Steven K., \& Roybal, Marsha M., April, 2014 